\title{
Endoplasmic reticulum stress: cell life and death decisions
}

\author{
Chunyan Xu, Beatrice Bailly-Maitre, and John C. Reed
}

The Burnham Institute for Medical Research, La Jolla, California, USA.

\begin{abstract}
Disturbances in the normal functions of the ER lead to an evolutionarily conserved cell stress response, the unfolded protein response, which is aimed initially at compensating for damage but can eventually trigger cell death if ER dysfunction is severe or prolonged. The mechanisms by which ER stress leads to cell death remain enigmatic, with multiple potential participants described but little clarity about which specific death effectors dominate in particular cellular contexts. Important roles for ER-initiated cell death pathways have been recognized for several diseases, including hypoxia, ischemia/reperfusion injury, neurodegeneration, heart disease, and diabetes.
\end{abstract}

\section{Introduction}

The ER fulfills multiple cellular functions (reviewed in refs. 1-4). The lumen of the ER is a unique environment, containing the highest concentration of $\mathrm{Ca}^{2+}$ within the cell because of active transport of calcium ions by $\mathrm{Ca}^{2+}$ ATPases. The lumen is an oxidative environment, critical for formation of disulfide bonds and proper folding of proteins destined for secretion or display on the cell surface. Because of its role in protein folding and transport, the ER is also rich in $\mathrm{Ca}^{2+}$-dependent molecular chaperones, such as Grp78, Grp94, and calreticulin, which stabilize protein folding intermediates (reviewed in refs. 1, 5-7).

Many disturbances, including those of cellular redox regulation, cause accumulation of unfolded proteins in the ER, triggering an evolutionarily conserved response, termed the unfolded protein response (UPR). Glucose deprivation also leads to ER stress, by interfering with $\mathrm{N}$-linked protein glycosylation. Aberrant $\mathrm{Ca}^{2+}$ regulation in the ER causes protein unfolding, because of the $\mathrm{Ca}^{2+}$ dependent nature of Grp78, Grp94, and calreticulin (6). Viral infection may also trigger the UPR, representing one of the ancient evolutionary pressures for linking ER stress to cell suicide in order to avoid spread of viruses. Further, because a certain amount of basal protein misfolding occurs in the ER, normally ameliorated by retrograde transport of misfolded proteins into the cytosol for proteasome-dependent degradation, situations that impair proteasome function can create a veritable protein traffic jam and can even cause inclusion body diseases associated with neurodegeneration.

The initial intent of the UPR is to adapt to the changing environment, and reestablish normal ER function. These adaptive mechanisms involve transcriptional programs that induce expression of genes that enhance the protein folding capacity of the ER, and promote ER-associated protein degradation to remove misfolded proteins. Translation of mRNAs is also initially inhibited, reducing the influx of new proteins into the ER for hours until mRNAs encoding UPR proteins are produced. When adaptation fails,

Nonstandard abbreviations used: $\mathrm{AD}$, Alzheimer disease; $\mathrm{A} \beta \mathrm{P}$, amyloid $\beta$-peptide; DED-L, death effector domain-like; eIF $2 \alpha$, eukaryotic initiation factor $2 \alpha ; \mathrm{Htt}$, Huntingtin; IP3, inositol triphosphate; IP3R, IP3 receptor; NOS, nitric oxide synthase; PD, Parkinson disease; PERK, PKR-like ER kinase; polyQ, polyglutamine; PS-1, presenilin-1; SERCA, sarcoplasmic/endoplasmic reticulum $\mathrm{Ca}^{2+}$ ATPase; UPR, unfolded protein response; XBP-1, X box protein-1.

Conflict of interest: The authors have declared that no conflict of interest exists.

Citation for this article: J. Clin. Invest. 115:2656-2664 (2005).

doi:10.1172/JCI26373.
ER-initiated pathways signal alarm by activating NF- $\mathrm{B}$, a transcription factor that induces expression of genes encoding mediators of host defense. Excessive and prolonged ER stress triggers cell suicide, usually in the form of apoptosis, representing a last resort of multicellular organisms to dispense of dysfunctional cells. Progress in understanding the mechanisms underlying these 3 phases of adaptation, alarm, and apoptosis has improved our knowledge of ER stress, and its role in disease.

\section{Adaptation to ER stress: mechanisms to restore homeostasis}

When unfolded proteins accumulate in the ER, resident chaperones become occupied, releasing transmembrane ER proteins involved in inducing the UPR. These proteins straddle ER membranes, with their N-terminus in the lumen of the ER and their C-terminus in the cytosol, providing a bridge that connects these 2 compartments. Normally, the N-termini of these transmembrane ER proteins are held by ER chaperone Grp78 (BiP), preventing their aggregation. But when misfolded proteins accumulate, Grp78 releases, allowing aggregation of these transmembrane signaling proteins, and launching the UPR. Among the critical transmembrane ER signaling proteins are PERK, Ire1, and ATF6 (Figure 1) (reviewed in refs. 1, 2, 8).

PERK (PKR-like ER kinase) is a Ser/Thr protein kinase, the catalytic domain of which shares substantial homology to other kinases of the eukaryotic initiation factor $2 \alpha(\operatorname{eIF} 2 \alpha)$ family $(9,10)$. Upon removal of Grp78, PERK oligomerizes in ER membranes, inducing its autophosphorylation and activating the kinase domain. PERK phosphorylates and inactivates eIF $2 \alpha$, thereby globally shutting off mRNA translation and reducing the protein load on the ER. However, certain mRNAs gain a selective advantage for translation under these conditions, including the mRNA encoding transcription factor ATF4. The ATF4 protein is a member of the bZIP family of transcription factors, which regulates the promoters of several genes implicated in the UPR. The importance of PERK-initiated signals for protection against ER stress has been documented by studies of perk ${ }^{-/-}$cells and of knock-in cells that express non-phosphorylatable eIF $2 \alpha(\mathrm{S} 51 \mathrm{~A})$, both of which display hypersensitivity to ER stress $(11,12)$.

Ire 1 similarly oligomerizes in ER membranes when released by Grp78. The Ire $1 \alpha$ protein is a type I transmembrane protein, which contains both a Ser/Thr kinase domain and an endoribonuclease domain; the latter processes an intron from $\mathrm{X}$ box protein-1 (XBP-1) 


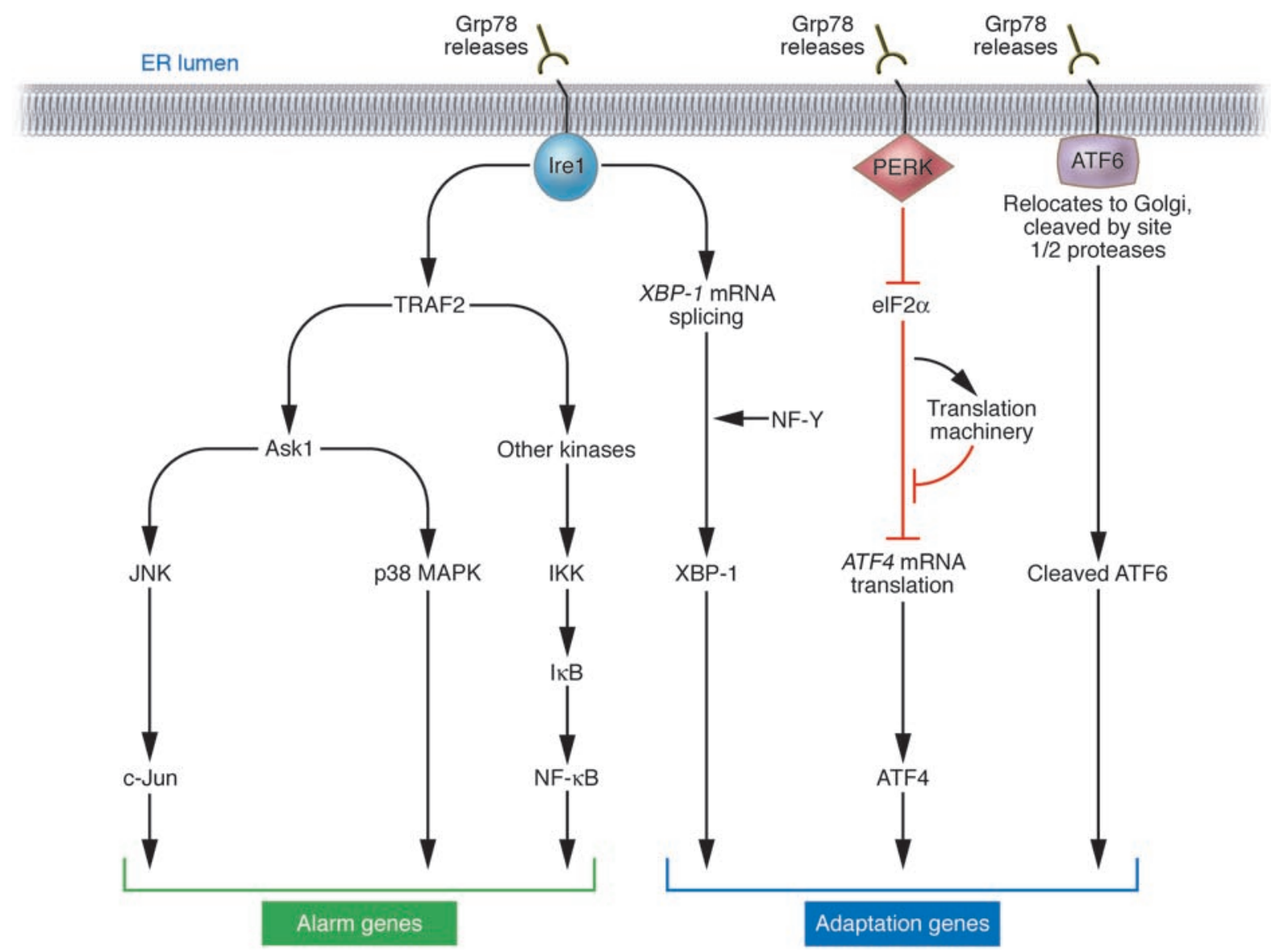

Figure 1

Signal transduction events associated with ER stress. Chaperone Grp78 binds the N-termini of Ire1, PERK, and ATF6, preventing their activation. Unfolded proteins in the ER cause Grp78 to release Ire1, PERK, and ATF6. Upon Grp78 release, Ire1 and PERK oligomerize in ER membranes. Oligomerized Ire1 binds TRAF2, signaling downstream kinases that activate NF-кB and c-Jun (AP-1), causing expression of genes associated with host defense (alarm). The intrinsic ribonuclease activity of Ire1 also results in production of XBP-1, a transcription factor that induces expression of genes involved in restoring protein folding or degrading unfolded proteins. Oligomerization of PERK activates its intrinsic kinase activity, resulting in phosphorylation of elF2 $\alpha$ and suppression of mRNA translation. Under these conditions, only selected mRNAs, including ATF4, are translated. ATF4 induces expression of genes involved in restoring ER homeostasis. Release of Grp78 from ATF6 allows this protein to translocate to the Golgi apparatus for proteolytic processing to release active ATF6, which controls expression of UPR genes.

mRNA, rendering it competent for translation to produce the 41-kDa XBP-1 protein, a bZIP-family transcription factor. XBP-1 binds to promoters of several genes involved in retrograde transport of misfolded proteins from ER to cytosol and in ER-induced protein degradation (reviewed in ref. 8). XBP-1 heterodimerizes with protein NF-Y and binds at least 2 types of cis-acting elements in gene promoters, including the ER stress enhancer (ERSE) and unfolded protein response element (UPRE) (13). Ablation of Ire $1 \alpha$ in mice produces an embryonic lethal phenotype. Fibroblasts from Ire $1 \alpha^{-/-}$embryos are defective in activation of UPRE-driven reporter genes, thus showing a cause-and-effect linkage of Ire $1 \alpha$ to this cis-acting element (14).

Release of Grp78 from the N-terminus of ATF6 triggers a different mechanism of protein activation, compared with PERK and Ire1. Instead of oligomerizing, release of Grp78 frees ATF6 to translocate to the Golgi apparatus, where resident proteases (site 1 and site 2 protease) cleave ATF6 at a juxtamembrane site, releasing this transcription factor into the cytosol and allowing it to migrate into the nucleus to regulate gene expression (15). ATF6 collaborates with Ire1, where ATF6 induces transcription to increase XBP-1 mRNA, and Ire1's endoribonuclease activity then processes that mRNA so that XBP-1 protein is produced.

\section{Sounding the alarm in response to ER stress: NF- $\kappa$ B activation mechanisms}

Given the massive glycoprotein production associated with many viral infections, it is not surprising that ER stress activates some of the same signal transduction pathways associated with innate immunity. In this regard, Ire 1 shares in common with many members of the TNF receptor family the ability to bind adapter protein TRAF2. TRAF2 is an E3 ligase that binds Ubc13, resulting in non-canonical polyubiquitination of substrates involving lysine 63 rather than the canonical lysine 48 as a linking site (16). TRAF2 activates protein kinases implicated in immunity and inflammation, including Ask1, which activates JNK, and kinases linked to $\mathrm{NF}-\kappa \mathrm{B}$ activation. Also recruited to Ire 1 is the $\mathrm{c}$-Jun N-terminal inhibitory kinase (JIK), responsible for posttranslational modification of components of the Ire $1 \alpha /$ TRAF2/Ask 1 complex $(17,18)$. 


\section{Apoptosis induced by ER stress: so many mechanisms, so little clarity}

The adaptive responses to misfolded proteins in the ER provide protection from cell death, inasmuch as gene transfer-mediated overexpression of Grp78 or protein-disulfide isomerase (PDI) reduces cell death induced by oxidative stress, $\mathrm{Ca}^{2+}$ disturbances, and hypoxia $(19,20)$. However, when protein misfolding is persistent or excessive, ER stress triggers cell death, typically apoptosis. Several mechanisms, described below, have been proposed for linking the distressed ER to cell death (Figure 2), including direct activation of proteases, kinases, transcription factors, and Bcl-2-family proteins and their modulators.

Proteases. Caspases are required for apoptosis, and certain members of this family of cysteine proteases associate with the ER (reviewed in ref. 21). In rodents, caspase-12 associates with activated Ire1, resulting in proteolytic processing of caspase-12. Mice lacking caspase-12 genes display partial resistance to pharmacological inducers of ER stress, such as tunicamycin (inhibitor of $\mathrm{N}$-linked protein glycosylation) and thapsigargin (inhibitor of sarcoplasmic/endoplasmic reticulum $\mathrm{Ca}^{2+}$ ATPases [SERCAs], which pump Ca ${ }^{2+}$ into the ER) (22). Because proteolytic activity has been difficult to demonstrate for caspase-12 (23), it is unknown whether the proteolytic processing of caspase- 12 that occurs during ER stress results in its activation. Also, the mechanisms responsible for proteolysis of caspase-12 may be indirect, involving calpains activated by $\mathrm{Ca}^{2+}$ released in the vicinity of the ER (24), instead of an induced proximity mechanism where oligomers of Ire 1 provide a scaffold for clustering caspase- 12 zymogens. Caspase-7 also may activate caspase- 12 by translocating from cytosol to ER (25). However, the relevance of caspase-12 to ER-induced apoptosis has been questioned because of an absence of caspase- 12 in most humans. In this regard, the ancestral human CASPASE-12 gene is disrupted by a termination codon and thus is inactive (26). For persons with hereditary polymorphisms that leave the open reading frame intact (estimated at $1 \%$ of African populations), caspase- 12 operates as a trans-dominant inhibitor of proinflammatory caspases, lacking conserved residues required for catalytic activity (23).

Human caspase-4, one of the closest paralogs of rodent caspase-12, may associate with ER (27), raising the possibility that this protease can perform the functions normally ascribed to rodent caspase- 12 in the context of ER stress. But caspase- 4 belongs to the group of proinflammatory caspases responsible for proteolytic activation of cytokines, rather than the apoptotic caspases. Nevertheless, small interfering RNA-mediated knock down of caspase- 4 in human neuroblastoma cells partially reduces cell death caused by the ER stress inducers thapsigargin and amyloid $\beta$-peptide (A $\beta \mathrm{P})$, but not inducers of mitochondria-dependent cell death (e.g., UV irradiation, DNA-damaging drugs). However, caspase- 4 knock down in HeLa cells had little effect on apoptosis induced by ER stress, implying that the relevance of this protease to ER stress is tissue-specific.

The ER resident protein Bap31 contains 3 predicted transmembrane domains, followed by a leucine zipper and a death effector domain-like (DED-L) region that associates with certain isoforms of procaspase-8 in the cytosol (28). Bap31 can display either pro- or antiapoptotic phenotypes, depending on whether its cytosolic tail is removed by cleavage by caspases. Overexpression of full-length Bap31 blocks apoptosis induced by anti-Fas antibody and cycloheximide, while expression of the truncated $20-\mathrm{kDa}$ protein induces apoptosis $(29,30)$. A mutant of Bap31 in which the caspase- 8 cleavage site was mutated suppressed Fas-induced apoptosis (29). Since proximal steps in Fas signaling were not blocked by mutant Bap31, this suggests that the ER participates as an intermediary in death receptor-induced apoptosis in some cells.

The DED-L domain of Bap31 also binds a homologous DED-L domain in BAR, another ER-associated apoptosis regulator (31). The $52-\mathrm{kDa}$ BAR protein contains a RING domain that binds ubiquitin-conjugating enzymes, followed by an $\alpha$-helical region that binds Bcl-2 and Bcl- $x_{\mathrm{L}}$, the DED-L domain, and a C-terminal membrane-anchoring domain (32). Like the DED-L domain of Bap31, the DED-L domain of BAR binds caspase-8, sequestering it and thwarting apoptosis induction initiated by TNF/Fas-family death receptors $(32,33)$. BAR also binds the apoptosis regulators Hip and Hippi, which contain DED-L domains homologous to those found in BAR and Bap31. Hip associates with Huntingtin (Htt), the protein implicated in Huntington disease that causes degeneration of neurons containing Htt polyglutamine (polyQ) expansions (34). Hippi is a DED-L domain-containing Hip-interacting protein that binds procaspase- 8 . Htt with polyQ expansion has reduced affinity for Hip compared with the normal Htt protein, a circumstance under which it has been proposed that Hip is free to bind Hippi and trigger caspase-8 activation (35). Interactions of BAR, Bap31, Hip, and Hippi deserve further investigation on a number of fronts, including whether these proteins represent substrates for the $\mathrm{E} 3$ ligase activity of BAR, elucidation of their agonistic and antagonistic relations among each other, and evaluation of effects of these protein interactions on nonapoptotic functions of $\mathrm{Htt}$ and its interacting proteins.

The ability of BAR and Bap31 to bind procaspase- 8 prompts speculation that perhaps these ER proteins could promote rather than inhibit caspase- 8 activation, if induced to aggregate in ER membranes, thereby constituting a novel ER-associated "apoptosome." If so, then the parallel ability of BAR and Bap31 to bind $\mathrm{Bcl}-2$ and $\mathrm{Bcl}-\mathrm{x}_{\mathrm{L}}$ through domains separate from the DED-L domain might supply a mechanism for preventing caspase activation, providing a long-sought analogy to the paradigm for caspase regulation seen in Caenorhabditis elegans, where the Bcl-2 ortholog Ced9 binds caspase activator Ced4, preventing activation of Ced3 protease (36).

Kinases. The kinase Ask1 has been implicated in apoptosis induction in the context of signaling by TNF-family receptors (reviewed in ref. 37). During ER stress, Ask1 is recruited to oligomerized Ire1 complexes containing TRAF2, activating this kinase and causing downstream activation of JNK and p38 MAPK. Consistent with a key role for Ask1 in apoptosis induced by ER stress, studies of ask $1^{-/-}$ neurons subjected to inducers of ER stress indicate a requirement for this kinase for JNK activation and cell death (38). The downstream death effectors of Ask1 are not clear. The kinase pathway initiated by Ask 1 leads to JNK activation, and JNK-mediated phosphorylation activates the proapoptotic protein Bim (39-41), while inhibiting the antiapoptotic protein Bcl-2 (42).

Thus, Ire 1 plays roles in all 3 of the ER responses to unfolded proteins (adaptation, alarm, and apoptosis), through its actions upon XBP-1 (adaptation), TRAF2 (alarm [NF-кB]), and apoptosis effectors caspase-12 and Ask1. How these 3 functions of Ire 1 are integrated remains unclear.

The protein tyrosine kinase c-Abl can translocate from the ER surface to mitochondria in response to ER stress (43). Moreover, a functional role for c-Abl has been suggested by studies of $c-A b l^{-/-}$fibroblasts, which display resistance to cell death induced 
by $\mathrm{Ca}^{2+}$ ionophores, brefeldin A, and tunicamycin (43). How c-Abl promotes apoptosis is unknown at present.

Transcription factors. CHOP (GADD153) is a member of the $\mathrm{C} / \mathrm{EBP}$ family of bZIP transcription factors, and its expression is induced to high levels by ER stress (reviewed in ref. 44). The chop gene promoter contains binding sites for all of the major inducers of the UPR, including ATF4, ATF6, and XBP-1, and these transcription factors play causative roles in inducing chop gene transcription. Cause-and-effect roles in chop gene induction have been demonstrated for signaling molecules involved in ER stress by genetic manipulation of mice, showing that perk ${ }^{-/-}$and atf4 $4^{-/}$cells and $\operatorname{eIF} 2 \alpha(\mathrm{S} 51 \mathrm{~A})$ knock-in cells fail to induce chop during ER stress $(11,12,45)$. Cross-talk between the PERK/eIF2 $\alpha$ pathway and the Ire1/TRAF2/Ask1 pathway may also enhance CHOP activity at a posttranscriptional level, given that Ask1 activates both JNK and p38 MAPKs, and phosphorylation of the CHOP protein on serine 78 and serine 81 by p38 MAPKs increases its transcriptional and apoptotic activity (46). In addition to the aforementioned regulators, upstream activators of chop also include ATF2, which is induced by hypoxia and which is required for chop induction during amino acid starvation (47).

Overexpression of CHOP protein induces apoptosis, through a Bcl-2-inhibitable mechanism $(48,49)$. Moreover, chop $^{-/-}$mice are resistant to kidney damage induced by tunicamycin and to brain injury resulting from cerebral artery occlusion, demonstrating a role for CHOP in cell destruction when ER stress is involved (48, 50). How CHOP induces apoptosis is unclear. CHOP forms heterodimers with other C/EBP-family transcription factors via bZIPdomain interactions, which suppresses their binding to C/EBP sites in DNA, while promoting binding to alternative DNA sequences for target gene activation (51). Consequently, $\mathrm{CHOP}$ inhibits expression of genes responsive to C/EBP-family transcription factors, while enhancing expression of other genes containing the consensus motif 5'-(A/G)(A/G)(A/G)TGCAAT(A/C)CCC-3'. One relevant target may be $b c l-2$, whose expression is suppressed by $\mathrm{CHOP}$, at least in some cellular contexts (49). CHOP may also have nontranscriptional actions, still poorly defined (44). While capable of inducing apoptosis and contributing to cell death in several scenarios involving ER stress, CHOP is not essential for cell death induced by ER stress, as demonstrated by the observation that perk ${ }^{-/}$and eIF $2 \alpha($ S51A) knock-in cells are hypersensitive to ER stress-induced apoptosis but fail to induce chop gene expression $(12,45)$.

Scotin is another ER-targeted apoptosis inducer (52). The gene encoding Scotin is a direct target of p53, suggesting a way to link DNA damage to ER-mediated cell death mechanisms.

Bcl-2-family proteins and their modulators. Association of certain Bcl-2/ Bax-family proteins with ER membranes dates back to the initial discovery of Bcl-2 (53). Though better known for their actions upon mitochondria, $\mathrm{Bcl}-2 / \mathrm{Bax}$-family proteins also integrate into $\mathrm{ER}$

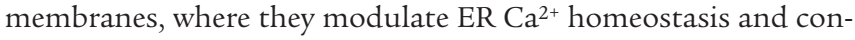
trol cell death induced by ER stress agents, including tunicamycin, brefeldin A (an inhibitor of ER-Golgi transport), thapsigargin, and oxidants (reviewed in ref. 54). Experiments in which the normal C-terminal transmembrane domain of $\mathrm{Bcl}-2$ was swapped with membrane-targeting domains from $\mathrm{ER}$ resident proteins suggested that Bcl-2 targeted exclusively to the ER (as opposed to both ER and mitochondria) is more restricted in its antiapoptotic actions, suppressing cell death induced by ER stress agents and by c-Myc. Recent findings that apoptosis induced by c-Myc may be attributable to its induction of Bim suggest that ER-targeted Bcl-2 may sequester this $\mathrm{BH} 3$-only protein, preventing it from interacting with other members of the Bcl-2/Bax family (55).

Spike is a BH3-only protein anchored in the ER (56). The BH3like domain of Spike is required for apoptosis induction, but dimerization partners among $\mathrm{Bcl}-2 / \mathrm{Bax}$-family proteins have yet to be found. Several other Bcl-2/Bax-family proteins reside at least in part in association with or integrated into ER membranes, with some, such as the antiapoptotic protein $\mathrm{Mcl}-1$ and proapoptotic $\mathrm{Bik}$, found predominantly in the $\mathrm{ER}(57,58)$. Given the preferences of certain $\mathrm{BH} 3$ domains for interactions with particular members of the Bcl-2/Bax family (59), it seems likely that a network of interactions among a subset of this family of apoptosis regulators takes place on ER membranes, the functional consequences of which are not yet fully understood. Recently, expression of at least 1 of the Bcl-2/Bax-family genes was linked to ER stress. The BH3-only protein Puma is induced by tunicamycin and thapsigargin in a p53-independent manner, with $\mathrm{Puma}^{-/}$cells showing resistance to apoptosis induced by ER stress (60).

The BI-1 protein contains 6 transmembrane domains, resides in the ER (61), interacts functionally or physically with Bcl-2-family members, and is induced by hypoxia (62). This protein blocks cell death induced by oxidative stress in yeast, plants, and animals (63). Mice lacking BI-1 display increased sensitivity to tunicamycin-induced kidney damage and to stroke injury, implying a role for BI-1 in protection from insults known to trigger ER stress. In cultured cells, overexpression of BI-1 selectively reduces, while BI-1 deficiency selectively increases, sensitivity to cell death induced by agents that trigger ER stress, while having far less effect on apoptosis induced by agents that trigger cell death pathways linked to mitochondria (intrinsic pathway) or TNF/Fas-family death receptors (extrinsic pathway) (64). BI-1 associates with the antiapoptotic proteins $\mathrm{Bcl}-2$ and $\mathrm{Bcl}-\mathrm{x}_{\mathrm{L}}$, but not proapoptotic $\mathrm{Bax}$ and Bak (61). Nevertheless, BI-1 inhibits cell death induced by Bax overexpression, in animal cells, plants, and yeast.

The ER protein Bap31 lacks homology with Bcl-2/Bax-family proteins and contains no recognizable $\mathrm{BH} 3$ dimerization domain, but it binds Bcl-2 and $\mathrm{Bcl}-\mathrm{x}_{\mathrm{L}}$ and regulates apoptosis. BAR also binds $\mathrm{Bcl}-2$ and $\mathrm{Bcl}-\mathrm{x}_{\mathrm{L}}$, and the responsible domain is required for BAR-mediated suppression of cell death (32). Interestingly, BAR is capable of suppressing Bax-induced death of yeast, implying caspase-independent functions for this protein, given that yeast lack bona fide caspases. This suggests that, mechanistically, BAR may share something in common with Bcl-2 and BI-1, which also suppress Bax-induced killing of yeast.

Other apoptosis regulators. Given that mitochondria release apoptogenic proteins into the cytosol, the ER might use similar mechanisms for linking ER stress to cell death. In insect cells, at least one example has been uncovered of a protein, called Jafrac2, that is normally sequestered in the ER but is released into the cytosol during apoptosis induced by certain stimuli (65). Like most proteins imported into the ER, the $\mathrm{N}$-terminal leader peptide of Jafrac2 is removed by proteolysis. This proteolytic processing exposes an IAP-binding motif in Jafrac2, poising it to attack antiapoptotic IAPfamily proteins upon accessing the cytosol, thereby freeing caspases. It remains to be determined whether examples of apoptogenic protein release from the ER of mammalian cells will be discovered.

\section{$\mathrm{Ca}^{2+}$ and apoptosis induced by ER stress}

Release of $\mathrm{Ca}^{2+}$ from the ER plays critical roles in cellular signaling mediated by the second messengers inositol triphosphate 


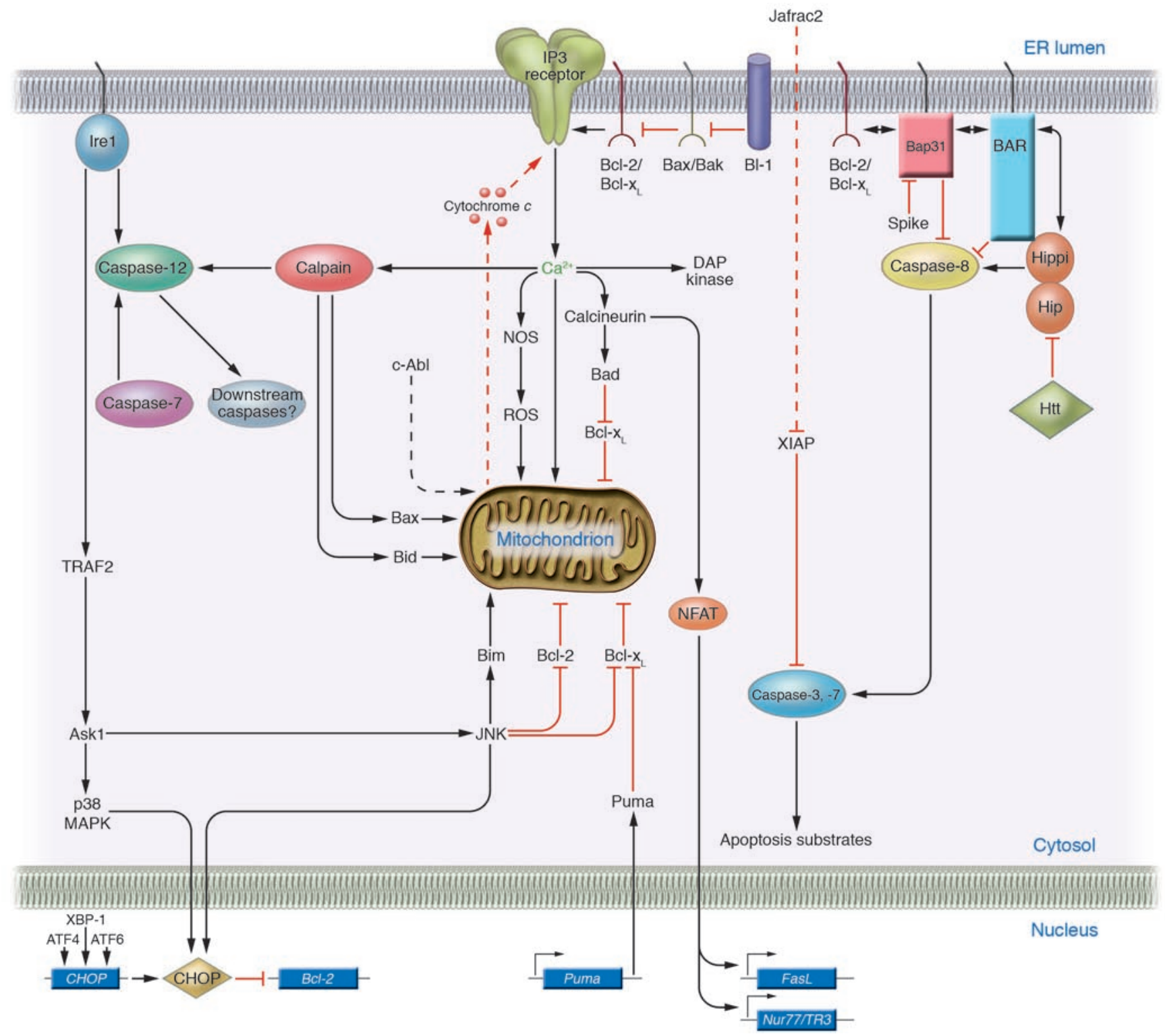

Figure 2

Cell death mechanisms induced by ER stress. Several of the proposed pathways linking ER stress to cell death are depicted. Dashed lines indicate protein translocation events (c-Abl, Jafrac2). The mitochondrial permeability transition pore complex, which is $\mathrm{Ca}^{2+-}$ sensitive, is not shown in the diagram. See the text for additional details.

(IP3) and cytosolic ADP-ribose and other regulators via effects on IP3 receptors (IP3Rs) and ryanodine receptors $(66,67)$. Opposing these gated $\mathrm{Ca}^{2+}$ channels are the SERCA-family proteins, $\mathrm{Ca}^{2+}$ ATPases that pump $\mathrm{Ca}^{2+}$ into the ER, which are regulated by phosphorylation and interactions with other proteins (e.g., phospholamban and sarcolipin). Various stimuli that cause the ER to dump $\mathrm{Ca}^{2+}$ precipitate cell death, including hypoxia, oxidants, stimulators of IP3 production, and pharmacological antagonists of SERCA. The downstream effectors of $\mathrm{Ca}^{2+}$-induced cell death are potentially myriad and could minimally include (a) induction of mitochondrial permeability transition, induced upon entry of excessive amounts of $\mathrm{Ca}^{2+}$ into the matrix of mitochondria (68, 69); (b) local activation near the ER of calpains, a family of $\mathrm{Ca}^{2+}$ dependent cysteine proteases implicated in pathological cell death
$(70,71)$, whose substrates include Bax and Bid (which are activated) (72-74), Bcl-2 and Bcl- $x_{\mathrm{L}}$ (which are inhibited), and several caspases (reviewed in ref. 4); (c) alterations in $\mathrm{Ca}^{2+}$-dependent phospholipid scramblases, which alter membrane biology to promote apoptosis or necrosis, including transferring phosphatidylserine to the outer leaflet of the plasma membrane (a signal for clearance of cells by phagocytosis) and transferring cardiolipin from the inner to outer membrane of mitochondria (a signal for targeted insertion of proapoptotic Bcl-2-family proteins Bid and Bax into membranes) (75-77); (d) $\mathrm{Ca}^{2+} /$ calmodulin-mediated activation of the protein phosphatase calcineurin, which dephosphorylates the proapoptotic protein Bad, allowing it to dimerize with and antagonize Bcl- $\mathrm{x}_{\mathrm{L}}$ (78), and which dephosphorylates NFAT-family transcription factors, allowing entry into the nucleus and trans- 
activation of proapoptotic genes encoding FasL and Nur77/TR3 (79); (e) stimulation of $\mathrm{Ca}^{2+}$-sensitive isoforms of nitric oxide synthase (NOS), exacerbating oxidative stress (reviewed in ref. 5); (f) activation of death-associated protein kinase (DAP kinase) and its relative DRP-1, which contain calmodulin-binding domains (reviewed in ref. 80); (g) activation of $\mathrm{Ca}^{2+}$-sensitive mitochondrial fission protein DRP-1 (81), which has been implicated in Baxinduced release of cytochrome $c$ from mitochondria; and possibly (h) alterations in the $\mathrm{Ca}^{2+}$-binding protein TCTP (fortilin), a putative modulator of antiapoptotic $\mathrm{Bcl}-2 / \mathrm{Bax}$-family proteins such as Mcl-1 (82). In addition, ectopic expression of the proapoptotic mammalian protein Bak in yeast induces cell death through a calnexin-dependent pathway, correlating with Bak binding to this $\mathrm{Ca}^{2+}$-dependent ER chaperone (83).

A role for Bcl-2 in modulating intracellular $\mathrm{Ca}^{2+}$ was first demonstrated over a decade ago (84), but only recently have clues about the mechanisms involved begun to emerge. Based on data from a variety of techniques, it appears that overexpression of antiapoptotic proteins $\mathrm{Bcl}-2$ and $\mathrm{Bcl}-\mathrm{x}_{\mathrm{L}}$ lowers the basal amounts of $\mathrm{Ca}^{2+}$ in the ER, because of increased leakage of $\mathrm{Ca}^{2+}$ under resting conditions. The consequence of this is that upon exposure to stimuli that precipitously dump $\mathrm{Ca}^{2+}$ from internal stores, less $\mathrm{Ca}^{2+}$ enters the cytosol, resulting in lower peak concentrations of cytosolic $\mathrm{Ca}^{2+}$ and less overall cytosolic $\mathrm{Ca}^{2+}$ accumulation (54, 85-88). Downstream, less $\mathrm{Ca}^{2+}$ enters mitochondria, which possibly explains the inhibition of mitochondrial depolarization and the suppression of cytochrome $c$ release. Like overexpression of $\mathrm{Bcl}-2$ or Bcl- $\mathrm{x}_{\mathrm{L}}$, ablation of the expression of proapoptotic Bax and Bak also reduces basal $\mathrm{Ca}^{2+}$ in the ER, implying a role for these proapoptotic proteins in setting cellular $\mathrm{ER} \mathrm{Ca}^{2+}$ concentrations (89). Interestingly, $\mathrm{Bcl}-2$ remains competent in its ability to reduce ER $\mathrm{Ca}^{2+}$, even in cells lacking Bax and Bak, implying that Bcl-2 operates downstream of $\mathrm{Bax} / \mathrm{Bak}$ with respect to $\mathrm{ER} \mathrm{Ca}^{2+}$ regulation, unlike the situation with mitochondria-dependent cell death, where genetic evidence indicates that $\mathrm{Bcl}-\mathrm{x}_{\mathrm{L}}$ and $\mathrm{Bcl}-2$ function upstream of Bax/Bak (90).

Attempts to establish whether these changes in ER $\mathrm{Ca}^{2+}$ handling mediated by $\mathrm{Bcl}-2 / \mathrm{Bax}$-family proteins are causally linked to cell death regulation have failed to provide firm answers, but supporting evidence has been obtained from a variety of experimental approaches, including genetic manipulations of $\mathrm{Ca}^{2+}$-regulating proteins in the $\mathrm{ER}(88,89,91,92)$.

Because several $\mathrm{Bcl}-2 / \mathrm{Bax}$-family proteins share structural similarity with the pore-forming domains of bacterial toxins, they may function as ion channels, thus explaining the ability of Bcl-2/ Bax-family proteins to modulate $\mathrm{ER} \mathrm{Ca}^{2+}$ (reviewed in ref. 93). However, mutations designed to impair the putative pore-forming regions of $\mathrm{Bcl}-2$ do not affect its ability to regulate $\mathrm{ER} \mathrm{Ca}^{2+}(94)$; this suggests alternative mechanisms. In this regard, Bcl-2 was reported to bind IP3Rs several years ago (95), and recently Bcl-2 has been implicated in regulating IP3R activity (96). IP3R knock down inhibits the ability of $\mathrm{Bcl}-2$ to promote leakage of $\mathrm{Ca}^{2+}$ from the ER, suggesting that $\mathrm{Bcl}-2$ relies on IP3Rs to reduce luminal ER $\mathrm{Ca}^{2+}$. The mechanism by which Bcl-2 modulates IP3Rs has yet to be defined, particularly the issue of whether this is a direct effect of these proteins on IP3Rs or an indirect effect on unidentified IP3R-interacting proteins present in ER membranes. Interestingly, cytochrome $c$, an apoptosis-inducing protein released from mitochondria, binds IP3Rs and induces $\mathrm{Ca}^{2+}$ release from ER, thereby triggering ER stress (97) and providing another potential link between IP3Rs and cell death regulation. Also, reduction in or ablation of expression of certain IP3Rs (e.g., IP3R1 and IP3R3) decreases sensitivity of some types of cells (e.g., lymphocytes, neurons) to apoptosis (98-100), suggesting further links between $\mathrm{Ca}^{2+}$ dysregulation by IP3Rs and apoptosis induction.

Curiously, the antiapoptotic protein BI-1 also regulates ER Ca ${ }^{2+}$ homeostasis in a manner analogous to that of Bcl-2 and $\mathrm{Bcl}-\mathrm{x}_{\mathrm{L}}$. Overexpression of $\mathrm{BI}-1$ reduces basal ER $\mathrm{Ca}^{2+}$ concentrations, while ablation of the genes encoding BI-1 increases amounts of thapsigarginreleasable $\mathrm{Ca}^{2+}$ from internal stores (64). Since BI-1 associates with $\mathrm{Bcl}-2$ and $\mathrm{Bcl}-\mathrm{x}_{\mathrm{L}}$ in ER membranes (61), it will be interesting to determine whether BI-1 also interacts with and regulates IP3Rs.

The truncated Bap31, resembling the caspase-cleavage product, induces $\mathrm{Ca}^{2+}$ efflux from the ER and induces apoptosis (30), providing further correlative connections between modulation of ER $\mathrm{Ca}^{2+}$ dynamics and cell death regulation. Interestingly, Bap31 was reported to bind an ER-associated putative ion channel called A4, but the relevance of this protein interaction to regulation of ER $\mathrm{Ca}^{2+}$ remains unclear (101).

\section{ER stress and diseases}

ER stress is associated with a range of diseases, including ischemia/ reperfusion injury, neurodegeneration, and diabetes (reviewed in ref. 44), making ER stress a probable instigator of pathological cell death and dysfunction.

ER stress and neurodegeneration. $\mathrm{A} \beta \mathrm{P}$ is a proteolytic product of amyloid $\beta$-precursor protein that is causally associated with Alzheimer disease (AD). Mice lacking caspase-12 are partially resistant to apoptosis induced by exposure to $\mathrm{A} \beta \mathrm{P}(22)$, raising the possibility of a functional link between ER stress and A $\beta$ P-induced toxicity. Mutant versions of the A $\beta$ P-interacting protein presenilin-1 (PS-1), previously associated with AD, interfere with the UPR (102) and may render neurons more susceptible to cell death induced by ER stress (103). The brains of mice harboring AD mutants of PS-1 also have increased CHOP protein (104). Interestingly, PS-1 induces cleavage of Ire $1 \alpha$, releasing the cytosolic domain to translocate to the nucleus, suggesting further interactions between molecules involved in AD and ER stress responses (105).

Hereditary mutations in the ER-associated E3 ubiquitin ligase Parkin have also been associated with ER stress-induced cell death and are found in patients with familial Parkinson disease (PD) $(106,107)$. Overexpression of wild-type Parkin suppresses cell death induced by several ER stress-inducing agents, and by $\alpha$-synuclein, the principal component of pathological Lewy bodies seen in PD (107). Parkin expression is induced by ER stress, suggesting a role for it in adaptation to ER stress, presumably functioning in the ER-associated protein degradation pathway to clear misfolded proteins.

Neurodegenerative diseases associated with inclusion body formation and protein aggregation have also been linked to ER stress, including amyotrophic lateral sclerosis, PD, Huntington disease, and others (reviewed in refs. 44, 108). Htt variants with polyQ expansions, for example, induce classical signal transduction events associated with the UPR and cause proteolytic processing of caspase-12 (109), as well as cause global reductions in proteasome activity (110). Thus, by exhausting the cytosolic protein degradation machinery, inclusion body diseases probably cause a back-up of misfolded proteins in the ER, triggering ER stress.

ER stress and ischemia/reperfusion injury. Reduced blood flow resulting from arterial occlusion or cardiac arrest results in tissue 
hypoxia and hypoglycemia, which cause protein misfolding and ER stress. Reperfusion of the affected tissues then triggers oxidative stress, with production of $\mathrm{NO}$, and other reactive oxygen species that result in protein misfolding. $\mathrm{NO}$ and other reactive molecules also may modify oxidizable residues (cysteines) in ER-associated $\mathrm{Ca}^{2+}$ channels, including ryanodine receptors and SERCAs, causing $\mathrm{ER} \mathrm{Ca}^{2+}$ depletion, yet another cause of protein misfolding.

Brain ischemia/reperfusion injury activates the PERK/eIF2 $\alpha$ pathway and induces chop expression in rodents $(111,112)$. Moreover, $c h o p^{-/-}$mice suffer less tissue loss after stroke, implying a causal role for this mediator of ER stress in neuronal cell death (113). NO, a known mediator of brain injury during stroke, induces chop expression in cultured neurons. Furthermore, a NOS inhibitor is protective in a rodent model of brain ischemia (114), and mice lacking the gene encoding iNOS display decreased sensitivity to brain ischemia (115), suggesting a causal role for this ER stress inducer in stroke damage.

A role for the antiapoptotic protein BI-1 in protection from cerebral ischemia has been demonstrated by studies of $b i-1^{-/-}$mice, which suffer larger infarcts following cerebral artery occlusion (64). Given that hypoxia has been implicated in bi-1 gene induction (62), these findings imply a role for endogenous $b i-1$ in survival of cells traumatized by ER stress.

ER stress and heart disease. The role of ER stress in heart disease has not been extensively studied, but Ask1 kinase activity increases in mice following myocardial infarction or aortic constriction, and ask $1^{-/}$mice showed reduced cardiomyocyte apoptosis, in addition to better preservation of left ventricular function, compared with wild-type animals (116).

ER stress in diabetes. Pancreatic $\beta$ cells have a well-developed ER, reflecting their role in secreting large amounts of insulin and various glycoproteins. This function of $\beta$ cells may explain why mice lacking PERK are susceptible to diabetes, showing apoptosis of their $\beta$ cells and progressive hyperglycemia with aging (117). Moreover, PERK gene mutations in association with infant-onset diabetes occur in humans with the autosomal recessive disorder Wolcott-Rallison syndrome (118). At autopsy, these patients show massive $\beta$ cell loss, resembling the pathology of perk ${ }^{-/-}$mice. Similarly, eIF $2 \alpha($ S51A) knock-in mice suffer from $\beta$ cell depletion, which begins in utero, suggesting a more rapid course than that in perk $^{-/-}$mice (12). The failure of perk ${ }^{-/-}$to phenocopy eIF2 $\alpha(\mathrm{S} 51 \mathrm{~A})$ raises the possibility that other kinases besides PERK inhibit eIF2 $\alpha$ during ER stress. Pancreatic $\beta$ cell apoptosis induced by NO, a mediator of inflammation relevant to autoimmune diabetes, is CHOP-dependent, further implicating ER stress as an instigator of $\beta$ cell death (50). Also, in rodent models of diabetes caused by a nonsecreted insulin mutant, homozygous deletion of chop delays disease onset (119), implying a role for this gene in $\beta$ cell depletion in vivo. Recently, $x b p-1^{+/-}$heterozygous mice have been shown to be more sensitive to diabetes caused by obesity and high-fat diet (120). The underlying mechanism is related to the requirement of XBP-1 for dampening of JNK activation caused by ER stress, which correlates with phosphorylation of IRS- 1 and reduced tyrosine phosphorylation of IRS-1 in insulin-stimulated cells. Interestingly, signs of ER stress were found in liver and adipose tissue of obese mice and mice fed high-fat diets, indicating that the metabolic abnormalities associated with obesity and unhealthy diets cause ER stress in vivo.

Other cells that secrete proteins in large quantities may also be at risk for ER stress-induced apoptosis. For example, studies of perk ${ }^{-/-}$ mice indicate a requirement for differentiation (or survival) of plasma cells, known for their production of immunoglobulins (121).

Therapentic targets. Several mediators of ER-initiated cell death are candidates for drug discovery efforts, though some are better validated than others. Gene ablation studies in mice argue that agents inhibiting Ask1 and CHOP are attractive, because mice lacking these genes are phenotypically normal but exhibit reduced sensitivity to cell death induced by ER stressors, such as stroke and polyQ-expanded proteins associated with neurodegeneration (reviewed in refs. 38, 44). Presumably Ask 1 is also responsible for the hyperactivity of JNK associated with insulin resistance in the context of ER stress caused by high-fat diet. Ask1 theoretically could be attacked by small molecules targeting the ATP-binding site of the kinase domain, analogous to other kinase inhibitors recently approved for other indications. The CHOP protein may be difficult to attack with small-molecule drugs. However, since p38 MAPK augments CHOP activity, small-molecule antagonists of this kinase currently in development for inflammatory diseases might find utility as cytoprotective agents in clinical scenarios involving ER stress. Also, c-Abl inhibitors such as imatinib (Gleevec) could be examined for cytoprotective activity in ischemic and degenerative diseases, given recent evidence that c-Abl may relay death signals from ER to mitochondria (43).

Compounds that augment the PERK/eIF2 $\alpha$ pathway may also protect against cell death by ER stress. Indeed, a recent screen for inhibitors of neuronal death induced by tunicamycin identified compounds that suppress protein phosphatases responsible for dephosphorylation of eIF $2 \alpha$ on serine 51 , thus increasing accumulation of phosphorylated eIF $2 \alpha$ and providing protection from apoptosis induced by several inducers of ER stress (122). Interestingly, the prototype compound characterized (Salubrinal) apparently is not an active-site inhibitor of the phosphatase but rather specifically disrupts complexes containing GADD35 and protein phosphatase-1 (PP1), thereby preventing GADD34-mediated targeting of PP1 onto substrate phospho-eIF $2 \alpha$.

It remains to be determined whether broad-spectrum inhibitors of caspase-family cell death proteases would preserve cell survival in the face of ER stress, given that culture experiments have shown that nonapoptotic cell death still occurs in the presence of compounds such as benzoyl-valinyl-alaninyl-aspartyl-fluoromethylketone (zVAD-fmk), at least when strong pharmacological inducers of ER stress are used (64). However, mice lacking various individual caspases, including caspase-1, caspase-2, and caspase-11 (a probable caspase- 4 or -5 ortholog), are resistant to stroke injury (123), a condition in which ER stress participates in the cell death mechanism.

Provided that side effects from vascular instability are not an issue, inhibitors of NOS are also attractive, since mice that lack iNOS show decreased sensitivity to brain ischemia and reduced CHOP expression (115). Finally, inhibitors of proapoptotic Bcl-2/Bax-family proteins that operate upon ER membranes could be useful for ameliorating tissue loss due to stimulators of ER stress.

\section{Conclusions}

ER stress has been implicated in several diseases, and pathways linking ER stress to cell death have been reported. The principal challenge with any strategy for blocking cell death caused by ER stress lies with the multitude of parallel pathways potentially leading to downstream cell death mechanisms. Thus, blocking only 1 cell death pathway emanating from the ER may be inadequate to 
preserve cell survival. Further studies of genes and gene products involved in ER-initiated cell death are needed to fully validate targets for drug discovery.

\section{Acknowledgments}

We thank J. Valois for manuscript preparation, M. Hanaii for artwork, and the California Breast Cancer Research Program, the Fondation pour la Recherche Médicale, and the NIH (grants NS047855 and AG15393) for their generous support.

Address correspondence to: John C. Reed, The Burnham Institute for Medical Research, 10901 North Torrey Pines Road, La Jolla, California 92037, USA. Phone: (858) 646-3140; Fax: (858) 6463194; E-mail: reedoffice@burnham.org.
1. Schroder, M., and Kaufman, R.J. 2005. ER stress and the unfolded protein response. Mutat. Res. 569:29-63.

2. Shen, X., Zhang, K., and Kaufman, R.J. 2004. The unfolded protein response: a stress signaling pathway of the endoplasmic reticulum [review]. J. Chem. Neuroanat. 28:79-92.

3. Rao, R.V., Ellerby, H.M., and Bredesen, D.E. 2004. Coupling endoplasmic reticulum stress to the cell death program. Cell Death Differ. 11:372-380.

4. Breckenridge, D.G., Germain, M., Mathai, J.P., Nguyen, M., and Shore, G.C. 2003. Regulation of apoptosis by endoplasmic reticulum pathways. Oncogene. 22:8608-8618.

5. Orrenius, S., Zhivotovsky, B., and Nicotera, P. 2003. Regulation of cell death: the calcium-apoptosis link [review]. Nat. Rev. Mol. Cell Biol. 4:552-565.

6. Ma, Y., and Hendershot, L.M. 2004. ER chaperone functions during normal and stress conditions. J. Chem. Neuroanat. 28:51-65.

7. Rizzuto, R., Duchen, M.R., and Pozzan, T. 2004 Flirting in little space: the ER/mitochondria $\mathrm{Ca} 2+$ liaison [review]. Sci. STKE. 2004:re1.

8. Rao, R.V., and Bredesen, D.E. 2004. Misfolded proteins, endoplasmic reticulum stress and neurodegeneration. Curr. Opin. Cell Biol. 16:653-662.

9. Shi, Y., et al. 1998. Identification and characterization of pancreatic eukaryotic initiation factor 2 alpha-subunit kinase, PEK, involved in translational control. Mol. Cell. Biol. 18:7499-7509.

10. Harding, H.P., Zhang, Y., and Ron, D. 1999. Protein translation and folding are coupled by an endoplasmic-reticulum-resident kinase. Nature. 397:271-274.

11. Harding, H.P., Zhang, Y., Bertolotti, A., Zeng, H., and Ron, D. 2000. Perk is essential for translational regulation and cell survival during the unfolded protein response. Mol. Cell. 5:897-904.

12. Scheuner, D., et al. 2001. Translational control is required for the unfolded protein response and in vivo glucose homeostasis. Mol. Cell. 7:1165-1176.

13. Yoshida, H., Matsui, T., Yamamoto, A., Okada, T., and Mori, K. 2001. XBP1 mRNA is induced by ATF6 and spliced by IRE1 in response to ER stress to produce a highly active transcription factor. Cell. 107:881-891.

14. Lee, K., et al. 2002. IRE1-mediated unconventional mRNA splicing and S2P-mediated ATF6 cleavage merge to regulate XBP1 in signaling the unfolded protein response. Genes Dev. 16:452-466.

15. Ye, J., et al. 2000. ER stress induces cleavage of membrane-bound ATF6 by the same proteases that process SREBPs. Mol. Cell. 6:1355-1364.

16. Habelhah, H., et al. 2004. Ubiquitination and translocation of TRAF2 is required for activation of JNK but not of $\mathrm{p} 38$ or NF-kappaB. EMBOJ. 23:322-332.

17. Urano, F., et al. 2000. Coupling of stress in the ER to activation of JNK protein kinases by transmembrane protein kinase IRE1. Science. 287:664-666.

18. Yoneda, T., et al. 2001. Activation of caspase-12, an endoplastic reticulum (ER) resident caspase, through tumor necrosis factor receptor-associated factor 2-dependent mechanism in response to the ER stress. J. Biol. Chem. 276:13935-13940.

19. Liu, H., et al. 1997. Endoplasmic reticulum chaperones GRP78 and calreticulin prevent oxidative stress, $\mathrm{Ca} 2+$ disturbances, and cell death in renal epithelial cells. J. Biol. Chem. 272:21751-21759.

20. Tanaka, S., Uehara, T., and Nomura, Y. 2000.
Up-regulation of protein-disulfide isomerase in response to hypoxia/brain ischemia and its protective effect against apoptotic cell death. J. Biol. Chem. 275:10388-10393.

21. Momoi, T. 2004. Caspases involved in ER stressmediated cell death. J. Chem. Neuroanat. 28:101-105.

22. Nakagawa, T., et al. 2000. Caspase- 12 mediates endoplasmic-reticulum-specific apoptosis and cytotoxicity by amyloid- $\beta$. Nature. 403:98-103.

23. Saleh, M., et al. 2004. Differential modulation of endotoxin responsiveness by human caspase- 12 polymorphisms. Nature. 429:75-79.

24. Nakagawa, T., and Yuan, J. 2000. Cross-talk between two cysteine protease families. Activation of caspase- 12 by calpain in apoptosis. J. Cell Biol. 150:887-894.

25. Rao, R.V., et al. 2001. Coupling endoplasmic reticulum stress to the cell death program: mechanism of caspase activation. J. Biol. Chem. 276:33869-33874.

26. Fischer, H., Koenig, U., Eckhart, L., and Tschachler, E. 2002. Human caspase-12 has acquired deleterious mutations. Biochem. Biophys. Res. Commun. 293:722-726.

27. Hitomi, J., et al. 2004. Involvement of caspase-4 in endoplasmic reticulum stress-induced apoptosis and Abeta-induced cell death. J. Cell Biol. 165:347-356.

28. Ng, F.W.H., et al. 1997. p28 Bap31, a Bcl-2/Bcl-X $\mathrm{L}^{-}$ and procaspase-8-associated protein in the endoplasmic reticulum. J. Cell Biol. 139:327-338.

29. Nguyen, M., Breckenridge, D.G., Ducret, A., and Shore, G.C. 2000. Caspase-resistant BAP31 inhibits fas-mediated apoptotic membrane fragmentation and release of cytochrome $\mathrm{c}$ from mitochondria. Mol. Cell. Biol. 20:6731-6740.

30. Breckenridge, D.G., Stojanovic, M., Marcellus, R., and Shore, G.C. 2003. Caspase cleavage product of BAP31 induces mitochondrial fission through endoplasmic reticulum calcium signals, enhancing cytochrome c release to the cytosol. J. Cell Biol. 160:1115-1127.

31. Roth, W., et al. 2003. Bifunctional apoptosis inhibitor (BAR) protects neurons from diverse cell death pathways. Cell Death Differ. 10:1178-1187.

32. Zhang, H., et al. 2000. BAR: an apoptosis regulator at the intersection of caspase and bcl-2 family proteins. Proc. Natl. Acad. Sci. U. S. A. 97:2597-2602.

33. Stegh, A.H., et al. 2002. Inactivation of caspase- 8 on mitochondria of Bcl- $\mathrm{x}_{\mathrm{L}}$ expressing MCF7-Fas cells. J. Biol. Chem. 277:4351-4360.

34. Kalchman, M.A., et al. 1997. HIP1, a human homologue of S. cerevisiae Sla2p, interacts with membrane-associated huntingtin in the brain. Nat. Genet. 16:44-53.

35. Gervais, F.G., et al. 2002. Recruitment and activation of caspase- 8 by the Huntingtin-interacting protein Hip-1 and a novel partner Hippi. Nat. Cell Biol. 4:95-105.

36. Hengartner, M.O. 2000. The biochemistry of apoptosis. Nature. 407:770-776.

37. Matsukawa, J., Matsuzawa, A., Takeda, K., and Ichijo, H. 2004. The ASK1-MAP kinase cascades in mammalian stress response. J. Biochem. (Tokyo). 136:261-265.

38. Nishitoh, H., et al. 2002. ASK1 is essential for endoplasmic reticulum stress-induced neuronal cell death triggered by expanded polyglutamine repeats. Genes Dev. 16:1345-1355.

39. Lei, K., and Davis, R.J. 2003. JNK phosphorylation of Bim-related members of the Bcl2 family induces Bax-dependent apoptosis. Proc. Natl. Acad. Sci. U.S. A.
100:2432-2437.

40. Putcha, G.V., et al. 2003. JNK-mediated BIM phosphorylation potentiates BAX-dependent apoptosis. Neuron. 38:899-914.

41. Luciano, F., et al. 2003. Phosphorylation of Bim-EL by Erk $1 / 2$ on serine 69 promotes its degradation via the proteasome pathway and regulates its proapoptotic function. Oncogene. 22:6785-6793.

42. Yamamoto, K., Ichijo, H., and Korsmeyer, S.J. 1999. BCL-2 is phosphorylated and inactivated by an ASK1/Jun N-terminal protein kinase pathway normally activated at G(2)/M. Mol. Cell. Biol. 19:8469-8478

43. Ito, Y., et al. 2001. Targeting of the c-Abl tyrosine kinase to mitochondria in endoplasmic reticulum stress-induced apoptosis. Mol. Cell. Biol. 21:6233-6242.

44. Oyadomari, S., and Mori, M. 2004. Roles of CHOP/ GADD153 in endoplasmic reticulum stress. Cell Death Differ. 11:381-389.

45. Harding, H.P., et al. 2003. An integrated stress response regulates amino acid metabolism and resistance to oxidative stress. Mol. Cell. 11:619-633.

46. Wang, X.Z., and Ron, D. 1996. Stress-induced phosphorylation and activation of the transcription factor CHOP (GADD153) by p38 MAP Kinase. Science. 272:1347-1349.

47. Bruhat, A., et al. 2000. Amino acids control mammalian gene transcription: activating transcription factor 2 is essential for the amino acid responsiveness of the CHOP promoter. Mol. Cell. Biol. 20:7192-7204.

48. Zinszner, H., et al. 1998. CHOP is implicated in programmed cell death in response to impaired function of the endoplasmic reticulum. Genes Dev. 12:982-995.

49. McCullough, K.D., Martindale, J.L., Klotz, L.O., Aw, T.Y., and Holbrook, N.J. 2001. Gadd153 sensitizes cells to endoplasmic reticulum stress by downregulating $\mathrm{Bcl} 2$ and perturbing the cellular redox state. Mol. Cell. Biol. 21:1249-1259.

50. Oyadomari, S., et al. 2001. Nitric oxide-induced apoptosis in pancreatic beta cells is mediated by the endoplasmic reticulum stress pathway. Proc. Natl. Acad. Sci. U. S. A. 98:10845-10850.

51. Ubeda, M., et al. 1996. Stress-induced binding of the transcriptional factor CHOP to a novel DNA control element. Mol. Cell. Biol. 16:1479-1489.

52. Bourdon, J.C., Renzing, J., Robertson, P.L., Fernandes, K.N., and Lane, D.P. 2002. Scotin, a novel p53-inducible proapoptotic protein located in the ER and the nuclear membrane. J. Cell Biol. 158:235-246.

53. Tsujimoto, Y., and Croce, C. 1986. Analysis of the structure, transcripts, and protein products of $\mathrm{Bcl}-2$, the gene involved in human follicular lymphoma. Proc. Natl. Acad. Sci. U. S. A. 83:5214-5218.

54. Thomenius, M.J., and Distelhorst, C.W. 2003. $\mathrm{Bcl}-2$ on the endoplasmic reticulum: protecting the mitochondria from a distance [review]. J. Cell Sci. 116:4493-4499.

55. Egle, A., Harris, A.W., Bouillet, P., and Cory, S. 2004. Bim is a suppressor of Myc-induced mouse B cell leukemia. Proc. Natl. Acad. Sci. U. S. A. 101:6164-6169.

56. Mund, T., Gewies, A., Schoenfeld, N., Bauer, M.K., and Grimm, S. 2003. Spike, a novel BH3-only protein, regulates apoptosis at the endoplasmic reticulum. FASEB J. 17:696-698.

57. Yang, T., Kozopas, K.M., and Craig, R.W. 1995. The 
intracellular distribution and pattern of expression of Mcl-1 overlap with, but are not identical to, those of Bcl-2. J. Cell Biol. 128:1173-1184.

58. Mathai, J.P., Germain, M., Marcellus, R.C., and Shore, G.C. 2002. Induction and endoplasmic reticulum location of BIK/NBK in response to apoptotic signaling by E1A and p53. Oncogene. 21:2534-2544.

59. Chen, L., et al. 2005. Differential targeting of prosurvival $\mathrm{Bcl}-2$ proteins by their $\mathrm{BH} 3$-only ligands allows complementary apoptotic function. Mol. Cell. 17:393-403.

60. Reimertz, C., Kogel, D., Rami, A., Chittenden, T., and Prehn, J.H. 2003. Gene expression during ER stress-induced apoptosis in neurons: induction of the $\mathrm{BH} 3$-only protein $\mathrm{Bbc} 3 / \mathrm{PUMA}$ and activation of the mitochondrial apoptosis pathway. J. Cell Biol. 162:587-597.

61. Xu, Q., and Reed, J.C. 1998. BAX inhibitor-1, a mammalian apoptosis suppressor identified by functional screening in yeast. Mol. Cell. 1:337-346.

62. Blais, J.D., et al. 2004. Activating transcription factor 4 is translationally regulated by hypoxic stress. Mol. Cell. Biol. 24:7469-7482.

63. Chae, H.-J., et al. 2003. Evolutionarily conserved cytoprotection provided by Bax Inhibitor-1 homologs from animals, plants, and yeast. Gene. 323:101-113.

64. Chae, H.-J., et al. 2004. BI-1 regulates an apoptosis pathway linked to endoplasmic reticulum stress. Mol. Cell. 15:355-366.

65. Tenev, T., Zachariou, A., Wilson, R., Paul, A., and Meier, P. 2002. Jafrac2 is an IAP antagonist that promotes cell death by liberating Dronc from DIAP1. EMBO J. 21:5118-5129.

66. Berridge, M.J., Lipp, P., and Bootman, M.D. 2000. The versatility and universality of calcium signaling [review]. Nat. Rev. Mol. Cell Biol. 1:11-21.

67. Benkusky, N.A., Farrell, E.F., and Valdivia, H.H. 2004. Ryanodine receptor channelopathies. Biochem. Biophys. Res. Commun. 322:1280-1285.

68. Bernardi, P. 1999. Mitochondrial transport of cations: channels, exchangers, and permeability transition [review]. Physiol. Rev. 79:1127-1155.

69. Kroemer, G., and Reed, J.C. 2000. Mitochondrial control of cell death [review]. Nat. Med. 6:513-519.

70. Chan, S.L., and Mattson, M.P. 1999. Caspase and calpain substrates: roles in synaptic plasticity and cell death [review]. J. Neurosci. Res. 58:167-190.

71. Huang, Y., and Wang, K.K. 2001. The calpain family and human disease. Trends Mol. Med. 7:355-362.

72. Wood, D.E., et al. 1998. Bax cleavage is mediated by calpain during drug-induced apoptosis. Oncogene. 17:1069-1078.

73. Wood, D.E., and Newcomb, E.W. 1999. Caspasedependent activation of calpain during druginduced apoptosis. J. Biol. Chem. 274:8309-8315.

74. Chen, M., et al. 2001. Bid is cleaved by calpain to an active fragment in vitro and during myocardial ischemia/reperfusion. J. Biol. Chem. 276:30724-30728.

75. McMillin, J.B., and Dowhan, W. 2002. Cardiolipin and apoptosis. Biochem. Biophys. Acta. 1585:97-107.

76. Lutter, M., et al. 2000. Cardiolipin provides specificity for targeting of tBid to mitochondria. Nat Cell Biol. 2:754-756

77. Kuwana, T., et al. 2002. Bid, bax, and lipids cooperate to form supramolecular openings in the outer mitochondrial membrane. Cell. 111:331-342.

78. Wang, H.G., et al. 1999. Ca2+-induced apoptosis through calcineurin dephosphorylation of BAD. Science. 284:339-343.

79. Youn, H., Sun, L., Prywes, R., and Liu, J. 1999. Apoptosis of $\mathrm{T}$ cells mediated by $\mathrm{Ca} 2+$-induced release of the transcription factor MEF2. Science. 286:790-793.

80. Shohat, G., Shani, G., Eisenstein, M., and Kimchi, A. 2002. The DAP-kinase family of proteins: study of a novel group of calcium-regulated death-promoting kinases [review]. Biochim. Biophys. Acta. 1600:45-50.

81. Szabadkai, G., et al. 2004. Drp-1-dependent division of the mitochondrial network blocks intra- organellar $\mathrm{Ca} 2+$ waves and protects against $\mathrm{Ca} 2+-$ mediated apoptosis. Mol. Cell. 16:59-68.

82. Liu, H., Peng, H.W., Cheng, Y.S., Yuan, H.S., and Yang-Yen, H.F. 2005. Stabilization and enhancement of the antiapoptotic activity of mcl-1 by TCTP. Mol. Cell. Biol. 25:3117-3126.

83. Torgler, C.N., et al. 1997. Expression of bak in S. pombe results in a lethality mediated through interaction with the calnexin homologue Cnx1. Cell Death Differ. 4:263-271.

84. Baffy, G., Miyashita, T., Williamson, J.R., and Reed, J.C. 1993. Apoptosis induced by withdrawal of interleukin-3 (IL-3) from an IL-3-dependent hematopoietic cell line is associated with repartitioning of intracellular calcium and is blocked by enforced $\mathrm{Bcl}-2$ oncoprotein production. J. Biol. Chem. 268:6511-6519.

85. He, H., Lam, M., McCormick, T.S., and Distelhorst, C.W. 1997. Maintenance of calcium homeostasis in the reticulum by Bcl-2. J. Cell Biol. 138:1219-1228.

86. Pinton, P., et al. 2000. Reduced loading of intracellular $\mathrm{Ca} 2+$ stores and downregulation of capacitative $\mathrm{Ca} 2+$ influx in Bcl-2-overexpressing cells. J. Cell Biol. 148:857-862.

87. Pinton, P., et al. 2001. The $\mathrm{Ca}^{2+}$ concentration of the endoplasmic reticulum is a key determinant of ceramide-induced apoptosis: significance for the molecular mechanism of Bcl-2 action. EMBOJ. 20:2690-2701

88. Demaurex, N., and Distelhorst, C. 2003. Cell biology: apoptosis. The calcium connection. Science. 300:65-67.

89. Scorrano, L., et al. 2003. BAX and BAK regulation of endoplasmic reticulum Ca2+: a control point for apoptosis. Science. 300:135-139.

90. Wei, M.C., et al. 2001. Proapoptotic BAX and BAK a requisite gateway to mitochondrial dysfunction and death. Science. 292:727-730

91. Nakamura, K., et al. 2000. Changes in endoplasmic reticulum luminal environment affect cell sensitivity to apoptosis. J. Cell Biol. 150:731-740.

92. Palmer, A.E., Jin, C., Reed, J.C., and Tsien, R.Y. 2004 $\mathrm{Bcl}-2$-mediated alterations in endoplasmic reticulum $\mathrm{Ca} 2+$ analyzed with an improved genetically encoded fluorescent sensor. Proc. Natl. Acad. Sci. U. S. A. 101:17404-17409.

93. Schendel, S., Montal, M., and Reed, J.C. 1998. Bcl-2 family proteins as ion-channels. Cell Death Differ. 5:372-380.

94. Chami, M., et al. 2004. Bcl-2 and Bax exert opposing effects on $\mathrm{Ca} 2+$ signaling, which do not depend on their putative pore-forming region. J. Biol. Chem. 279:54581-54589.

95. Kuo, T.H., et al. 1998. Modulation of endoplasmic reticulum calcium pump by $\mathrm{Bcl}-2$. Oncogene. 17:1903-1910

96. Oakes, S.A., et al. 2005. Proapoptotic BAX and BAK regulate the type 1 inositol trisphosphate receptor and calcium leak from the endoplasmic reticulum. Proc. Natl. Acad. Sci. U. S. A. 102:105-110.

97. Boehning, D., et al. 2003. Cytochrome c binds to inositol $(1,4,5)$ trisphosphate receptors, amplifying calcium-dependent apoptosis. Nat. Cell Biol. 5:1051-1061.

98. Jayaraman, T., and Marks, A.R. 1997. T cells deficient in inositol 1,4,5-trisphosphate receptor are resistant to apoptosis. Mol. Cell. Biol. 17:3005-3012.

99. Khan, A.A., et al. 1996. Lymphocyte apoptosis: mediation by increased type 3 inositol 1,4,5-trisphosphate receptor. Science. 273:503-507.

100. Blackshaw, S., et al. 2000. Type 3 inositol 1,4,5-trisphosphate receptor modulates cell death. FASEB J. 14:1375-1379.

101. Wang, B., et al. 2003. Uncleaved BAP31 in association with A4 protein at the endoplasmic reticulum is an inhibitor of Fas-initiated release of cytochrome c from mitochondria. J. Biol. Chem. 278:14461-14468.

102.Katayama, T., et al. 1999. Presenilin-1 mutations downregulate the signalling pathway of the unfolded-protein response. Nat. Cell Biol. 1:479-485.

103.Terro, F., et al. 2002. Neurons overexpressing mutant presenilin-1 are more sensitive to apoptosis induced by endoplasmic reticulum-Golgi stress. J. Neurosci. Res. 69:530-539.

104. Milhavet, O., et al. 2002. Involvement of Gadd153 in the pathogenic action of presenilin-1 mutations. J. Neurochem. 83:673-681.

105. Niwa, M., Sidrauski, C., Kaufman, R., and Walter, P. 1999. A role for presenilin-1 in nuclear accumulation of ire 1 fragments and induction of the mammalian unfolded protein response. Cell. 99:691-702.

106.Dawson, T.M., and Dawson, V.L. 2003. Rare genetic mutations shed light on the pathogenesis of Parkinson disease. J. Clin. Invest. 111:145-151. doi:10.1172/JCI200317575.

107. Takahashi, R., Imai, Y., Hattori, N., and Mizuno, Y. 2003. Parkin and endoplasmic reticulum stress. Ann. N. Y. Acad. Sci. 991:101-106.

108. Kakizuka, A. 1998. Protein precipitation: a common etiology in neurodegenerative disorders? Trends Genet. 14:396-402.

109. Kouroku, Y., et al. 2002. Polyglutamine aggregates stimulate ER stress signals and caspase-12 activation. Hum. Mol. Genet. 11:1505-1515.

110.Bence, N.F., Sampat, R.M., and Kopito, R.R. 2001. Impairment of the ubiquitin-proteasome system by protein aggregation. Science. 292:1552-1555

111.Kumar, R., et al. 2001. Brain ischemia and reperfusion activates the eukaryotic initiation factor 2alpha kinase, PERK. J. Neurochem. 77:1418-1421.

112.Paschen, W., Gissel, C., Linden, T., Althausen, S., and Doutheil, J. 1998. Activation of gadd153 expression through transient cerebral ischemia: evidence that ischemia causes endoplasmic reticulum dysfunction. Brain Res. Mol. Brain Res. 60:115-122.

113.Tajiri, S., et al. 2004. Ischemia-induced neuronal cell death is mediated by the endoplasmic reticulum stress pathway involving CHOP. Cell Death Differ. 11:403-415.

114.Kohno, K., Higuchi, T., Ohta, S., Kumon, Y., and Sakaki, S. 1997. Neuroprotective nitric oxide synthase inhibitor reduces intracellular calcium accumulation following transient global ischemia in the gerbil. Neurosci. Lett. 224:17-20.

115.Iadecola, C., Zhang, F., Casey, R., Nagayama, M., and Ross, M.E. 1997. Delayed reduction of ischemic brain injury and neurological deficits in mice lacking the inducible nitric oxide synthase gene. J. Neurosci. 17:9157-9164.

116.Yamaguchi, O., et al. 2003. Targeted deletion of apoptosis signal-regulating kinase 1 attenuates left ventricular remodeling. Proc. Natl. Acad. Sci. U. S. A. 100:15883-15888.

117. Harding, H.P., et al. 2001. Diabetes mellitus and exocrine pancreatic dysfunction in perk-/- mice reveals a role for translational control in secretory cell survival. Mol. Cell. 7:1153-1163.

118.Araki, E., Oyadomari, S., and Mori, M. 2003. Endoplasmic reticulum stress and diabetes mellitus. Intern. Med. 42:7-14

119. Oyadomari, S., et al. 2002. Targeted disruption of the Chop gene delays endoplasmic reticulum stressmediated diabetes. J. Clin. Invest. 109:525-532. doi:10.1172/JCI200214550.

120.Ozcan, U., et al. 2004. Endoplasmic reticulum stress links obesity, insulin action, and type 2 diabetes. Science. 306:457-461.

121.Zhang, K., et al. 2005. The unfolded protein response sensor IRE $1 \alpha$ is required at 2 distinct steps in B cell lymphopoiesis. J. Clin. Invest. 115:268-281. doi:10.1172/JCI200521848.

122. Boyce, M., et al. 2005. A selective inhibitor of eIF2alpha dephosphorylation protects cells from ER stress. Science. 307:935-939.

123.Yuan, J., and Yankner, B.A. 2000. Apoptosis in the nervous system. Nature. 407:802-809. 